\title{
An unbiased approach de-livers unexpected insight into torsin biology
}

\author{
Sarah M. Prophet ${ }^{1}$ and Christian Schlieker ${ }^{1,2}$ \\ 'Department of Molecular Biophysics and Biochemistry, Yale University, New Haven, Connecticut, USA. ²Department of Cell Biology, Yale School of Medicine, New Haven, Connecticut, USA.
}

\begin{abstract}
Mutations affecting the integrity of the essential torsin ATPase/cofactor system have been identified in a steadily increasing number of congenital disorders. Since most of these mutations affect brain function, much of the research has focused on deciphering disease etiology in the brain. However, torsin is expressed in a wide variety of nonneural tissues and is strictly conserved across species, including the lowest metazoans, suggesting that it plays roles extending beyond neurons. In this issue of the $J C I$, Shin et al. explored torsin function in the mammalian liver. The group reports major defects in hepatic lipid metabolism when the torsin system is compromised in mice. Remarkably, conditional deletion of either torsinA or its cofactor, lamina-associated polypeptide 1 (LAP1), resulted in fatty liver disease and steatohepatitis, likely from a secretion defect of VLDLs. This study considerably expands our understanding of torsin biology, while providing defined opportunities for future investigations of torsin function and dysfunction in human pathologies.
\end{abstract}

TorsinA and congenital disease Torsins are proteins in the superfamily of ATPases associated with diverse cellular activities $\left(\mathrm{AAA}^{+}\right)(1)$. All four torsins encoded by the human genome localize within the endoplasmic reticulum (ER) and nuclear envelope (NE) system. Despite being members of the $\mathrm{AAA}^{+}$ ATPase superfamily, torsins are highly unusual ATPases. Perhaps their greatest departure from canonical $\mathrm{AAA}^{+}$proteins is their unique mechanism of ATPase activation. The torsin active site lacks critical elements and is therefore unable to hydrolyze ATP in isolation (2). Instead, torsins rely on one of two transmembrane protein cofactors found within the ER/NE system to complement its active site $(2,3)$. The first cofactor, located within the inner nuclear membrane (INM), is lamina-associated polypeptide 1 (LAP1); the second is luminal domain- like LAP1 (LULL1), which remains in the peripheral ER (4).

TorsinA was originally identified in a positional cloning approach to identify the genetic lesion underlying one of most common forms of childhood-onset dystonia (DYT1 dystonia) (5). Dystonias are a group of neurological disorders that often manifest as prolonged involuntary muscle contractions causing twisting movements. Although some forms of dystonia arise from injuries or drug exposure, many cases are genetic (1). DYT1 dystonia is caused by a genetic lesion on chromosome 9 , which deletes three base pairs of TOR1A, the gene that produces torsinA.

Patients with DYT1 dystonia are heterozygous for a TOR1A mutation that results in a single glutamate deletion (torsin $\mathrm{A} \triangle \mathrm{E}$ ) (5), which interrupts cofactorstimulated ATPase activation of torsinA (2, 3). Studies in several distinct model systems

Related Article: p. 4885

Conflict of interest: The authors have declared that no conflict of interest exists.

Copyright: @ 2019, American Society for Clinical Investigation.

Reference information: / Clin Invest. 2019;129(11):4576-4579. https://doi.org/10.1172/JCI132442

reported that manipulation of torsin or its cofactor compromises $\mathrm{NE}$ integrity and nuclear transport (6-8). Since torsin $\mathrm{A} \triangle \mathrm{E}$ fails to effectively rescue NE-specific phenotypes in torsinA-knockout systems, this mutation likely represents torsinA loss of function $(6,8,9)$. Notably, loss-offunction mutations in LAP1 were recently identified in patients with neurological impairment and shortened lifespan, again underscoring the importance of the torsinLAP1 assembly in the NE (10).

\section{Torsin redundancy and tissue- specific consequences}

The torsinA DYT1-associated mutation specifically affects neural tissue because of the expression pattern and redundancy. In humans, there are four torsin proteins, with torsin $\mathrm{A}$ and torsinB sharing the most similarity. In mice, torsins share some functional redundancy at the NE (11), and torsin A and torsinB are expressed at similar levels in many nonneural tissues including liver (12). In adult and embryonic mouse neural tissue, torsin $\mathrm{A}$ is much more abundant than torsinB (12). Consequently, if an organism acquires the torsin $\mathrm{A} \Delta \mathrm{E}$ mutation, torsin $B$ is not abundant enough to compensate for any loss of function in neural tissue. Multiple studies suggest, however, that torsinB can accommodate the loss of torsin A in nonneural tissue (13).

Shin and colleagues present an interesting system that suggests torsinB cannot always compensate for torsinA loss of function in nonneural tissue (14). Many studies that manipulate torsin A in vivo have characterized the effect on neurons. Shin et al. determined the significance of torsinA manipulation in hepatocytes. This study is the first to our knowledge to demonstrate an effect on lipid metabolism in nonneural tissue without knocking down torsinB in a mammalian context. Given the equal expression levels and high degree of redundancy between torsin $\mathrm{A}$ and torsin $\mathrm{B}$, the significant con- 
A

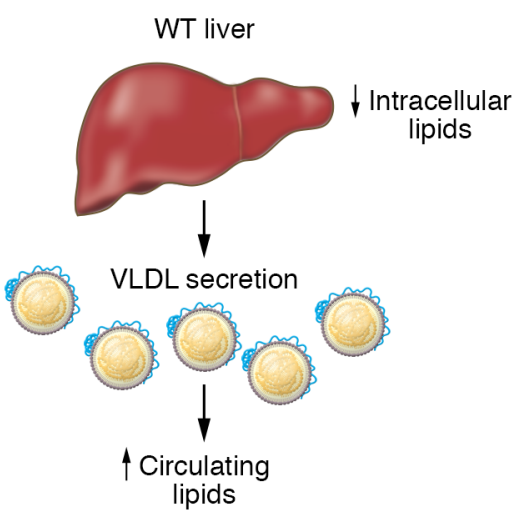

L-CKO/A-CKO liver

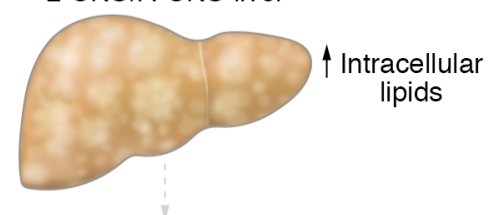

Impaired

VLDL secretion

$\downarrow$ Circulating

lipids

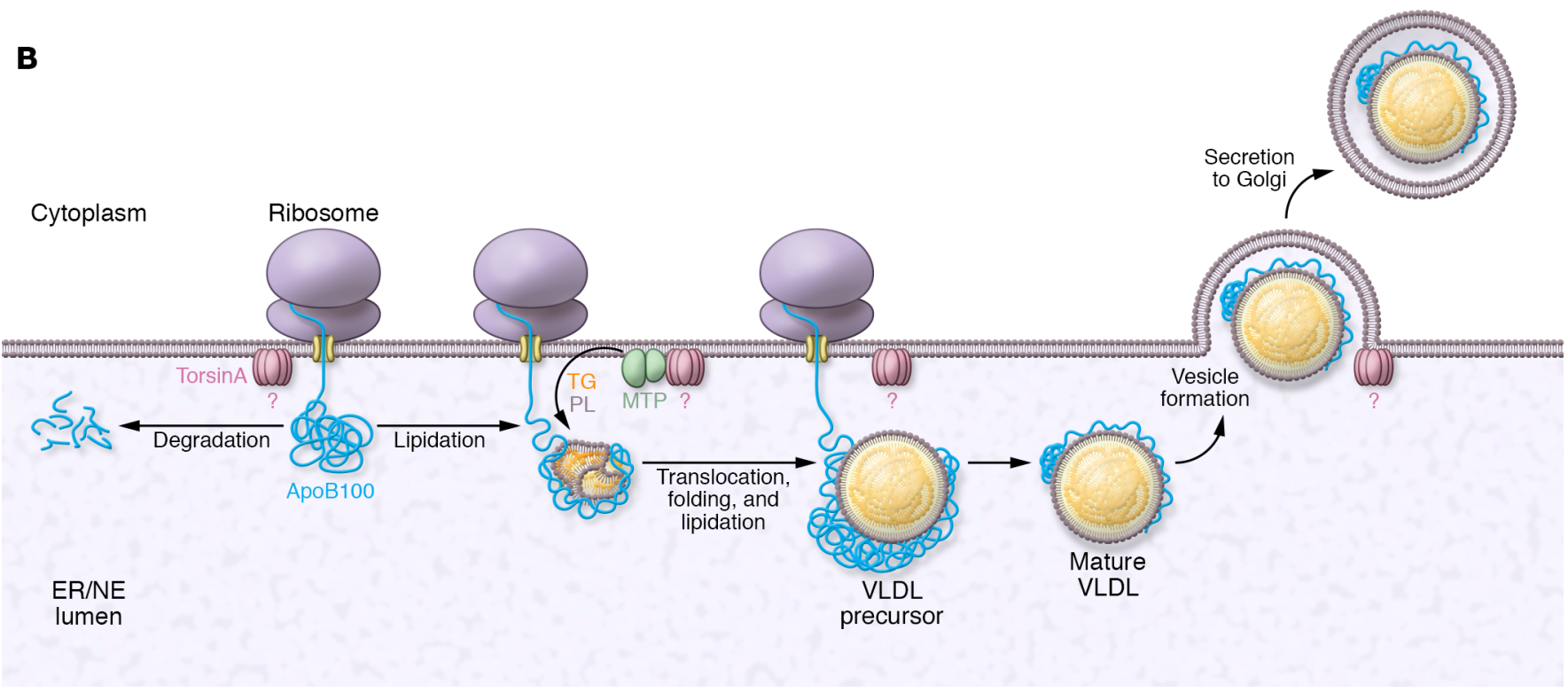

Figure 1. Possible roles for torsinA in the context of VLDL metabolism. (A) As WT livers secrete VLDLs, the VLDLs are released into the bloodstream and processed into smaller lipoproteins to deliver lipids to peripheral tissue. A-CKO and L-CKO livers retain lipids (steatosis) as a result of compromised VLDL secretion and can become whitish and enlarged. (B) Models for torsinA involvement in VLDL biogenesis. While apoB100 is translated into the ER, MTP aids in apoB100 lipidation by extracting phospholipids (PLs) and TGs from the ER. In the absence of lipidation, apoB100 is degraded. TorsinA may facilitate unlipidated apoB100 degradation or cooperate with MTP to facilitate apoB100 lipidation. Alternatively, torsinA may be involved in coordinating the translocation, folding, and lipidation of apoB100. Once the VLDL precursor grows to an appropriate size within the ER, the mature VLDL travels to the Golgi. VLDLs are packaged into specialized vesicles that may require torsinA for their formation.

sequences of knocking out torsinA in hepatocytes are striking (14).

\section{Torsins and lipid metabolism}

Shin et al. reported that the conditional deletion of torsinA (A-CKO) or LAP1 (L-CKO) in murine hepatocytes interfered with VLDL assembly and secretion, resulting in profound lipid dysregulation (14). The livers of A-CKO and L-CKO mice specifically exhibited excessive lipid retention (Figure 1A), a phenomenon that can lead to nonalcoholic fatty liver disease. The authors demonstrated that the nuclei of some hepatocytes from L-CKO mice are enlarged due to aberrantly localized intranuclear lipid droplets (LDs), which also increased in both number and size within the cytosol. In A-CKO hepatocytes, intranuclear LDs were absent. Instead, the cytosol and ER appeared tightly packed with LDs. Despite this difference in LD distribution, both A-CKO and L-CKO mouse livers retained cholesterol and triglycerides (TGs). Concomitantly, the mice showed significantly lower circulating cholesterol and TG levels (Figure 1A). Since A-CKO resulted in more severe phenotypes than did L-CKO, Shin et al. suggested that deletion of LAP1 in hepatocytes caused lipid retention by reducing torsinA activity at the INM (14).
The accumulation of lipids could result from disturbing any of the major processes in hepatic fatty acid (FA) metabolism, including the de novo synthesis of FAs, the generation of energy from FA oxidation, and the storage in LDs or secretion via VLDLs of FAs. Shin et al. observed no defects in lipogenesis or FA oxidation in L-CKO mice. Thus, considering that $\mathrm{L}$-CKO reduced circulating TGs, the retention of lipids seems likely to stem from a defect in VLDL secretion (Figure 1A). To investigate this, Shin et al. examined the secretion of a protein critical for VLDL biogenesis and integrity: apolipoprotein B100 (apoB100). The researchers used 
metabolic labeling to show that apoB100 was poorly secreted in both L-CKO and A-CKO mice (14). Importantly, this defect was specific to apoB100, as general protein secretion remained unaffected.

\section{Possible mechanisms and future directions}

What part of the apoB100 and VLDL secretion process could involve torsinA? As apoB100 is translated into the ER the chaperone microsomal TG transfer protein (MTP) adds lipids to apoB100 to form an initial lipid-poor VLDL precursor (15). If MTP fails to initiate lipidation, apoB100 undergoes proteasomal degradation (16). Shin et al. reported that in L-CKO and A-CKO livers, newly synthesized apoB100 levels were strongly reduced (14). Thus, torsinA could play a role in apoB100 lipidation alongside MTP (Figure 1B). Notably, a truncated version of torsin A was recently shown to deform lipid vesicles in vitro, a property that might facilitate mobilization of lipids from the lipid bilayer (17).

Alternatively, torsinA could be implicated in the coordination of apoB100 translation, folding, and degradation within the ER lumen (Figure 1B). Pulse-chase or imaging-based experiments that monitor the levels and localization of apoB100, along with inhibitors of ER-associated degradation machinery and the lysosomal pathway, could yield additional insight. It would also be interesting to test whether torsinA interacts with apoB100 during VLDL biogenesis in a manner that depends on lipid availability. Furthermore, whether LAP1 modulates the potential apoB100-VLDL interaction by controlling torsinA ATPase activity and oligomeric state would be exciting to investigate $(18,19)$.

Finally, torsin A could function during VLDL vesicular transport (Figure 1B). VLDLs are trafficked in vesicles unique from those that traffic bulk secreted proteins (20). If these specialized vesicles require torsin $\mathrm{A}$ for maturation, general protein secretion would appear normal, consistent with the Shin et al. findings (14).

In addition to the study by Shin et al., other recent work supports a potential role for torsin in lipid metabolism. Data from Grillet et al. suggest that the single torsin homolog in Drosophila melanogaster plays a critical role in promoting membrane proliferation during fly development (21). The researchers selectively expressed torsin in the neurons or muscles of flies otherwise devoid of the protein. However, neural or muscular torsin expression failed to rescue the pupal-stage lethality associated with torsin knockout (21). Instead, torsin expression within the larval fat body, an organ with many functional similarities to the liver, was necessary to suppress the knockout phenotype. Moreover, a connection between torsinA and lipid metabolism was recently proposed in a mouse model for primary dystonia (22). Thus, the emerging role of the torsin system in lipid metabolism, stemming from studies in various model systems, is likely evolutionarily conserved $(14,21,22)$.

The profound effect that Shin et al. observed upon LAP1 deletion raises a major question: How does losing torsinA activity at the INM interfere with VLDL biogenesis in the ER (20)? NE-localized LAP1 may affect lipid secretion indirectly by influencing torsin localization or by shifting the equilibrium of oligomeric torsin species. Another possibility is that the NE plays a yet-unknown role in the biogenesis of VLDL, which is consistent with our knowledge of diseases that affect lipid metabolism and are caused by mutations in INM proteins $(23,24)$. As outlined above, additional investigations will probe the testable hypotheses that were borne out by Shin et al., while also motivating a fresh look at lipid metabolism in patients with genetic lesions in LAP1 or torsinA. Concepts emerging from these future studies will enhance our understanding of torsin function and might provide important clues to further our understanding of torsin-related pathologies.

\section{Acknowledgments}

This work was supported by NIH grants (R01GM114401, to CS, and 5T32GM007223-44, to SMP). We sincerely apologize for any work not cited due to space restrictions.

Address correspondence to: Christian Schlieker, Yale University, Bass 229, 266 Whitney Avenue, New Haven Connecticut 06511, USA. Phone: 203.432.5035; Email: christian.schlieker@yale.edu.

1. Gonzalez-Alegre P. Advances in molecular and cell biology of dystonia: focus on torsinA.

Neurobiol Dis. 2019;127:233-241.
2. Brown RS, Zhao C, Chase AR, Wang J, Schlieker C. The mechanism of Torsin ATPase activation. Proc Natl Acad Sci US A. 2014;111(45):E4822-E4831.

3. Demircioglu FE, Sosa BA, Ingram J, Ploegh HL, Schwartz TU. Structures of TorsinA and its disease-mutant complexed with an activator reveal the molecular basis for primary dystonia. Elife. 2016;5:e17983.

4. Goodchild RE, Dauer WT. The $\mathrm{AAA}^{+}$protein torsinA interacts with a conserved domain present in LAP1 and a novel ER protein. JCell Biol. 2005;168(6):855-862.

5. Ozelius LJ, et al. The early-onset torsion dystonia gene (DYT1) encodes an ATP-binding protein. Nat Genet. 1997;17(1):40-48.

6. Goodchild RE, Kim CE, Dauer WT. Loss of the dystonia-associated protein torsinA selectively disrupts the neuronal nuclear envelope. Neuron. 2005;48(6):923-932.

7. VanGompel MJ, Nguyen KC, Hall DH, Dauer WT, Rose LS. A novel function for the Caenorhabditis elegans torsin OOC-5 in nucleoporin localization and nuclear import. Mol Biol Cell. 2015;26(9):1752-1763.

8. Pappas SS, Liang CC, Kim S, Rivera CO, Dauer WT. TorsinA dysfunction causes persistent neuronal nuclear pore defects. Hum Mol Genet. 2018;27(3):407-420.

9. Laudermilch E, Tsai PL, Graham M, Turner E, Zhao C, Schlieker C. Dissecting Torsin/cofactor function at the nuclear envelope: a genetic study. Mol Biol Cell. 2016;27(25):3964-3971.

10. Fichtman B, et al. Combined loss of LAP1B and LAP1C results in an early onset multisystemic nuclear envelopathy. Nat Commun. 2019;10(1):605.

11. Kim CE, Perez A, Perkins G, Ellisman MH, Dauer WT. A molecular mechanism underlying the neural-specific defect in torsinA mutant mice. Proc Natl Acad Sci U S A. 2010;107(21):9861-9866.

12. Jungwirth M, Dear ML, Brown P, Holbrook K, Goodchild R. Relative tissue expression of homologous torsinB correlates with the neuronal specific importance of DYT1 dystonia-associated torsinA. Hum Mol Genet. 2010;19(5):888-900.

13. Tanabe LM, Liang CC, Dauer WT. Neuronal nuclear membrane budding occurs during a developmental window modulated by torsin paralogs. Cell Rep. 2016;16(12):3322-3333.

14. Shin JY, et al. Nuclear envelope-localized torsinA-LAP1 complex regulates hepatic VLDL secretion and steatosis. JClin Invest. 2019;129(11):4885-4900.

15. Hussain MM, Shi J, Dreizen P. Microsomal triglyceride transfer protein and its role in apoB-lipoprotein assembly. J Lipid Res. 2003;44(1):22-32.

16. Ginsberg HN, Fisher EA. The ever-expanding role of degradation in the regulation of apolipoprotein B metabolism. JLipid Res. 2009;50 Suppl:S162-S166.

17. Demircioglu FE, et al. The $\mathrm{AAA}^{+}$ATPase TorsinA polymerizes into hollow helical tubes with 8.5 subunits per turn. Nat Commun. 2019;10(1):3262.

18. Zhao C, Brown RS, Chase AR, Eisele MR, Schlieker C. Regulation of Torsin ATPases 
by LAP1 and LULL1. Proc Natl Acad Sci U S A. 2013;110(17):E1545-E1554.

19. Chase AR, Laudermilch E, Wang J, Shigematsu $\mathrm{H}$, Yokoyama T, Schlieker C. Dynamic functional assembly of the Torsin $\mathrm{AAA}^{+}$ATPase and its modulation by LAP1. Mol Biol Cell. 2017;28(21):2765-2772.

20. Tiwari S, Siddiqi SA. Intracellular trafficking and secretion of VLDL. Arterioscler Thromb Vasc Biol. 2012;32(5):1079-1086.

21. Grillet M, et al. Torsins are essential regulators of cellular lipid metabolism. Dev Cell. 2016;38(3):235-247.

22. Cascalho A, et al. Inhibition of Lipin lipid phosphatase hyperactivity rescues TorsinA neurological disease. bioRxiv. https://doi.org/10.1101/606947.
Accessed September 11, 2019.

23. Dauer WT, Worman HJ. The nuclear envelope as a signaling node in development and disease. Dev Cell. 2009;17(5):626-638.

24. Jacquemyn J, Cascalho A, Goodchild RE. The ins and outs of endoplasmic reticulumcontrolled lipid biosynthesis. EMBO Rep. 2017;18(11):1905-1921. 